\title{
Eleven years of Dr D. S. Kothari Postdoctoral Fellowship scheme of the University Grants Commission
}

\author{
Rohit A. Kale, Subodh S. Khire, Sunil K. Hode, Dilip S. Joag* and Shridhar R. Gadre*
}

In 2007, the University Grants Commission (UGC), New Delhi, launched the Dr D. S. Kothari Postdoctoral Fellowship Scheme, guided by the Empowered Committee on Basic Scientific Research. This article provides a summary of the functioning and overall impact of this scheme after eleven years of operation along with future outlook.

Keywords: Academic institutions, basic science, fellowship, postdoctoral research.

POSTDOCTORAL research is an important academic training programme for young meritorious $\mathrm{Ph} \mathrm{D}$ degree holders to shape their future career. These trained postdoctoral Fellows are considered as potential teachers and researchers for academic institutions and industries. In the Indian university system, there was a dire need of such researchers in order to help academic institutions to achieve excellence. A national level postdoctoral research programme tailor-made for the university sector was considered for training necessary fresh $\mathrm{PhDs}$ in various science/engineering disciplines.

In 2006, the University Grants Commission (UGC), New Delhi and Ministry of Human Resource Development (MHRD), Government of India found that research and development activities are confined mainly to selected institutions in India. Hence it was considered necessary to strengthen research culture within the university system in the entire country. Therefore, MHRD constituted an Empowered Committee (EC) for Basic Scientific Research (BSR) to advise UGC (https://www.ugc.ac.in/ page/Basic-Scientific-Research.aspx) for enhancing the quality of research in universities. The first EC was constituted with the following members: M. M. Sharma, Goverdhan Mehta, S. P. Thyagarajan, Kota Harinarayan and P. Rama Rao. This EC under the chairmanship of Sharma initiated some new ventures in the Indian universities, such as Faculty Recharge Programme (FRP), Emeritus Fellowships and $\mathrm{Dr}$ D. S. Kothari Postdoctoral Fellowship (DSKPDF) scheme. Among these, DSKPDF, a flagship scheme of the UGC, was launched on 2 September 2007 and S. R. Gadre, Savitribai Phule Pune University (SPPU), Pune was identified as the National

The authors are in the UGC Dr D. S. Kothari Postdoctoral Fellowship Cell, Interdisciplinary School of Scientific Computing, Savitribai Phule Pune University, Pune 411 007, India.

*For correspondence. (e-mail: dsj@physics.unipune.ac.in; gadresr007@gmail.com)
Coordinator of the scheme by the EC. In 2010, on the recommendation of the EC, D. S. Joag (SPPU, Pune) was appointed as an Associate Coordinator. Execution of the scheme was entrusted to the SPPU, Pune. The website of DSKPDF (http://ugcdskpdf.unipune.ac.in/) was initially designed and developed by the Centre for Network Computing (CNC) personnel at SPPU, and is being maintained and upgraded by the staff of the DSKPDF cell. The cell staff developed a transparent and fast application processing mechanism. As a result, the DSKPDF Cell has become a part of fast-track, transparent and quality-based selection process (see Box 1) of the DSK Fellows. Initiation of the scheme required constant guidance and advice from the EC, whose members were always supportive.

In 2014, a new EC chaired by Anil D. Shahasrabudhe, with other eminent members being Ashok Jhunjhunwala, Syed A. Bari, Jawahar Lal Kaul and Vijay Bhatkar, replaced the earlier EC and also supported the scheme wholeheartedly.

Since the DSKPDF scheme has completed 11 years, this article presents its salient features and achievements of the scheme and helps create awareness about this unique academic endeavour of UGC and MHRD.

\section{Some salient features}

- DSKPDF covers a wide spectrum of sciences such as biological, chemical, physical, mathematical and earth and ocean sciences, accommodating medical and engineering sciences as well. The scheme is tenable only at the science departments of Indian universities and the respective constituent colleges as well as UGCfunded inter-university centres (for details of eligibility, see http://ugcdskpdf.unipune.ac.in/eligability.html).

- The DSKPDF scheme has an international flavour and is also open to candidates who are foreign nationals.

CURRENT SCIENCE, VOL. 118, NO. 3, 10 FEBRUARY 2020 
GENERAL ARTICLES

\section{Box 1. Evaluation procedure}

After the receipt of duly signed hard copy of DSKPDF application, a referee panel of five among the large pool of referees is selected based on the specialized topic of research of the candidate. Initially the application is sent for evaluation to three referees from the chosen panel. If three reports are not received in the stipulated time, reminders are sent to the referees. Else, the application is sent for evaluation to the fourth and fifth referees in succession.

Reviewers evaluate the application and assign a grade $(A / B / C / D)$. On the basis of the best three grades received and minimum selection criterion decided by the $\mathrm{EC}$, the qualifying candidates are recommended for the fellowship.

- The scheme is being run effectively by the DSKPDF Cell with the help of a highly distinguished pool of referees. The final result is prepared typically within 15 weeks from the receipt of the application hard copy and is placed in the ensuing EC meeting for approval.

- Currently, there are approximately 300 DSK Fellows working all over the country.

- The DSKPDF scheme runs in a flexible mode, with fast-track, on-line handling and decision-making platform.

- The administrative and financial matters are handled by the UGC-BSR section.

\section{Main highlights of the DSKPDF scheme}

The DSKPDF scheme has recently completed 11 years and here we present its highlights as well as current status.

\section{Success ratio of the scheme}

The DSKPDF Cell has received about 8200 applications for processing in the past 11 years, from which 2661 (32.4\%) candidates were selected as DSK Fellows based on rigorous evaluation by the referees followed by scrutiny by the EC (Table 1). After selection, about 2159 $(84.6 \%)$ candidates have so far joined the scheme, of which about 800 DSK Fellows were able to complete their tenure of three years (Table 2). The reason for this might be the permanent job offers to the DSK Fellows (many of them resigned due to this reason). It shows that one of the objectives of this scheme to serve as an academic bridge between post- $\mathrm{Ph} \mathrm{D}$ research and academic career has been achieved.

\section{Distribution of DSK Fellows}

Gender-wise: This quality-based selection also follows a fair gender-wise distribution (Table 3), with 956 (35.9\%) female DSK Fellows and 1705 (64.1\%) male DSK Fellows.
Table 1. The number of candidates and success ratio (\%) for selection (up to $81 \mathrm{st}$ selection list) under the Dr D. S. Kothari Postdoctoral Fellowship (DSKPDF) program (see text for details)

\begin{tabular}{lcc}
\hline Result & Number of candidates & Percentage \\
\hline Successful & 2661 & 32.4 \\
Unsuccessful & 5558 & 67.6 \\
Total & 8219 & 100.0 \\
\hline
\end{tabular}

Note: About $84.6 \%$ candidates have joined this scheme after selection.

Category-wise: The reservation policy of UGC is being followed with the merit of postdoctoral proposal during the selection process. Table 4 shows the category-wise distribution of DSK Fellows, which includes Open (1681), SC (279), ST (34), OBC (655) and PH (12) categories.

Workplace-wise: Figure 1 shows the workplace-wise distribution of DSK Fellows, who are evenly distributed in Central (41.07\%) as well as state universities $(39.88 \%)$. Also some autonomous institutions, mainly the Indian Institute of Science (IISc), Bengaluru; UGCDepartment of Atomic Energy (DAE) centres, etc. were the choice of about $19.05 \%$ DSK Fellows.

Geographical: The DSKPDF scheme has spread to several areas of the country. The success of any national scheme depends on its reach to all the geographical areas of the country. Figure 2 shows state-wise distribution of DSK Fellows.

\section{Publications as an outcome of this scheme}

The outcome of any research scheme may be measured by the quality of publications under the same. During the past 11 years DSK Fellows have published about 2160 research articles in international and 132 in national journals of high repute (Figure 3). The earlier data were not captured due to initial limitations of the system/nonfurnishing of data by the candidates. The number of publications is showing a steady increase as the DSK Fellows are continuously uploading their publications. The reputed journals in which the DSK Fellows have published include Angewandte Chemie International Edition (impact factor 13.4), Journal of the American Chemical Society (14.3), Nucleic Acids Research (11.6), Biomaterials (8.8), The Journal of Physical Chemistry Letters (8.7), Medicinal Research Reviews (8.2), ACS Applied Materials \& Interfaces (8), Antioxidants \& Redox Signaling (6.5), Plant Biotechnology Journal (6.3), Physical Review D (4.3), Computer Physics Communications (3.7) and Applied Physics Letters (3.4). These articles have been published mainly in the disciplines of biological sciences (825), 
Table 2. Discipline-wise publications under DSKPDF from 2012 to 2019

\begin{tabular}{lccc}
\hline Discipline & $\begin{array}{c}\text { No. of selected } \\
\text { candidates }\end{array}$ & $\begin{array}{c}\text { No. of DSK fellows } \\
\text { who joined }\end{array}$ & $\begin{array}{c}\text { Discipline-wise } \\
\text { publications }\end{array}$ \\
\hline Biological science (BL) & 1071 & 906 & 825 \\
Chemical science (CH) & 742 & 574 & 602 \\
Physical science (PH) & 475 & 388 & 593 \\
Mathematical science (MA) & 135 & 107 & 139 \\
Earth and ocean science (ES) & 93 & 71 & 40 \\
Engineering science (EN) & 81 & 60 & 57 \\
Medical science (ME) & 22 & 19 & 15 \\
Other sciences (OT) & 42 & 34 & 21 \\
Total & 2661 & 2159 & 2292 \\
\hline
\end{tabular}

Note: Only about 800 DSK Fellows have completed their tenure of three years.

Table 3. Gender-wise distribution (\%) of selected DSK fellows

\begin{tabular}{lcc}
\hline Gender & No. of DSK fellows & Percentage \\
\hline Female & 956 & 35.9 \\
Male & 1705 & 64.1 \\
Total & 2661 & 100.0 \\
\hline
\end{tabular}

Table 4. Category-wise DSKPDF applicants selected with respect to total applications received

\begin{tabular}{lccc}
\hline Category & $\begin{array}{c}\text { Total applications } \\
\text { received }\end{array}$ & $\begin{array}{c}\text { Total applicants } \\
\text { selected }\end{array}$ & $\begin{array}{c}\text { Selection } \\
(\%)\end{array}$ \\
\hline Open & 5240 & 1681 & 32.1 \\
SC & 821 & 279 & 34.0 \\
ST & 97 & 34 & 35.0 \\
OBC & 2028 & 655 & 32.3 \\
PH & 33 & 12 & 36.4 \\
Total & 8219 & 2661 & 32.4 \\
\hline
\end{tabular}

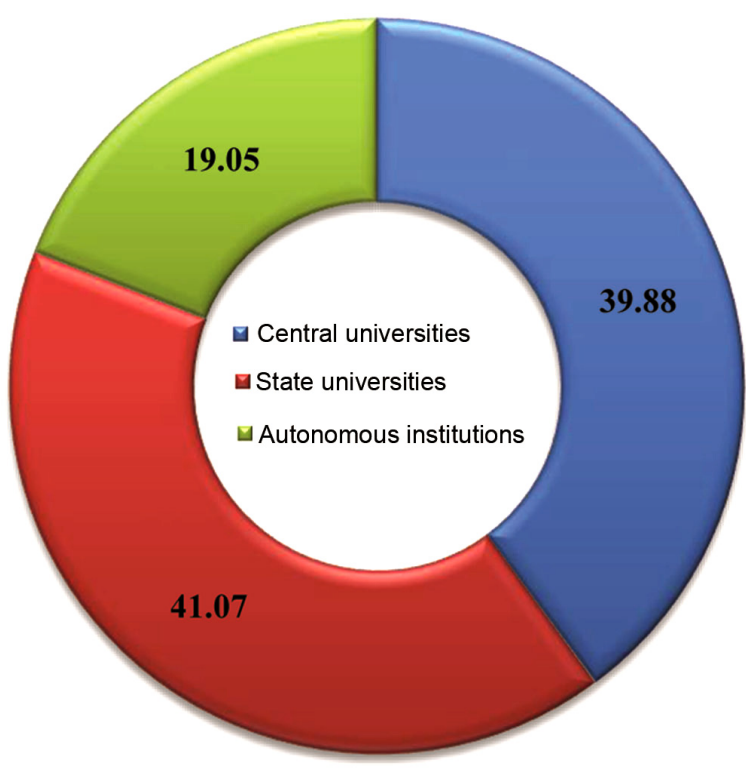

Figure 1. Workplace-wise distribution (\%) of Dr D. S. Kothari (DSK) Fellows (see text for details).

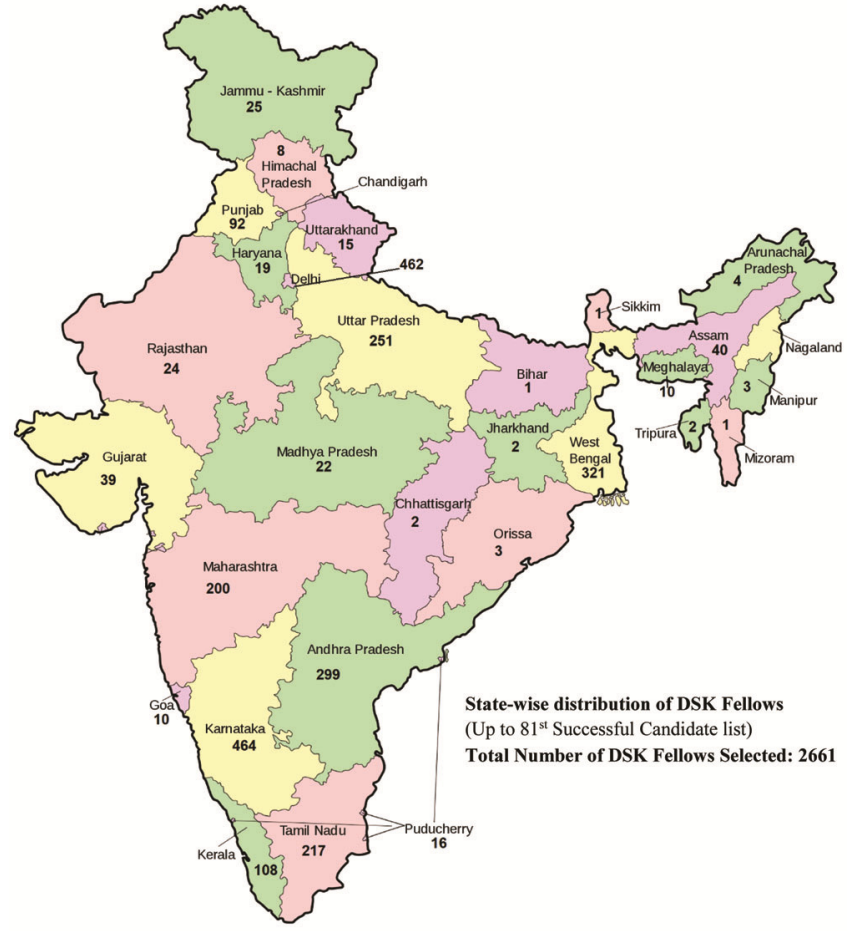

Figure 2. Geographical distribution of DSK Fellows.

chemical sciences (602) and physical sciences (593). Also, interestingly, about 139 research articles have been published in mathematical sciences by DSK Fellows (Figure 4).

\section{Feedback from Fellows, mentors and referees}

\section{Interaction meeting}

A regional monitoring and feedback meeting was held on 6 May 2015 at SPPU, in which the progress made by the DSK Fellows from Maharashtra and Goa region was evaluated. Several valuable suggestions were put forth by the subject experts present. Further, feedback was obtained from referees and DSK Fellows about the functional units of the DSKPDF scheme.

CURRENT SCIENCE, VOL. 118, NO. 3, 10 FEBRUARY 2020 


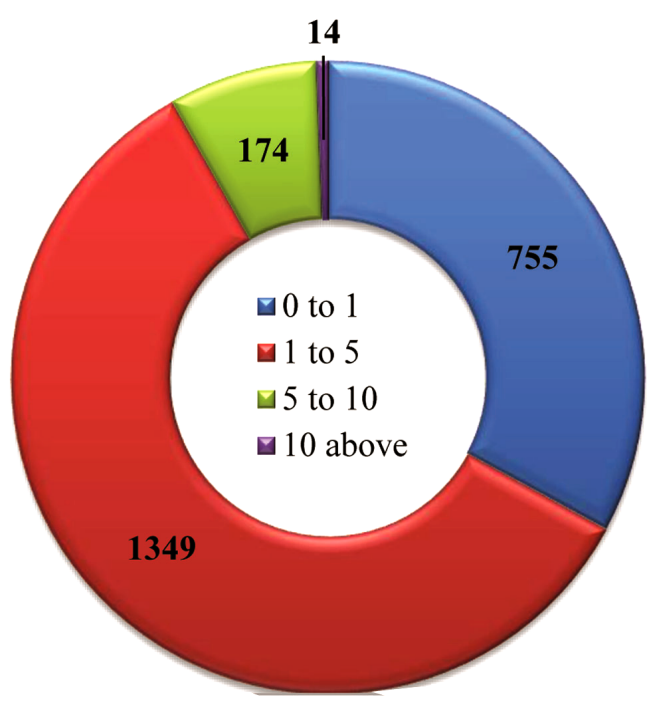

Figure 3. Impact factor-wise distribution of journals wherein DSK Fellows have published their research.

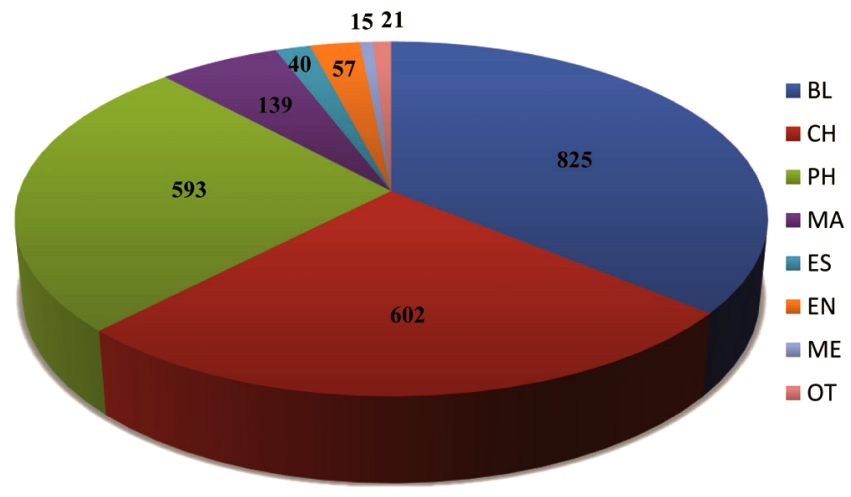

Figure 4. Subject-wise research papers published by DSK Fellows. See Table 2 for subject abbreviations.

\section{Representative views of mentors and visitors}

The DSKPDF Cell has obtained feedback from several mentors of DSK Fellows spread throughout the country. Some representative remarks are given below:

- 'D. S. Kothari Postdoctoral Fellowship is a wonderful program which contributes to the training of young people in the country. I give my strongest recommendation to continue with this program' (A. K. Sood, IISc).

- 'I am glad to note the system and progress of the premier DSKPDF scheme in the country. The coordi- nation of this scheme of the UGC by DSKPDF Cell has provided it much acceptability and sustainability. I congratulate to administrative staff and coordinators for their excellent work' (V. N. Rajasekharan Pillai, former Vice-Chairman, UGC).

- 'I am delighted to see the DSK Fellowship unit, and interact with staff. Over the years, the processing of application and the review process have become very efficient. I had a DSK Fellow a few years ago and she was the best postdoc I ever had! I do hope that this scheme gets strengthened in the coming years' (Uday Maitra, IISc).

\section{Overall impact of the scheme}

The DSKPDF Scheme of the UGC has an on-line, fasttrack, transparent and quality based process for selecting DSK Fellows. The selection is mainly based on the evaluation done by eminent referees. The gender-wise, reservation-wise, region-wise fair distribution of the selected Fellows is evident from the statistics. The benefits of the PDF culture in the career of young researchers in the country are immense. The usefulness of the DSKPDF scheme has been highlighted by several mentors as well as Fellows across the country. P. Balaram, former Director, IISc, Bengaluru has summarized the impact as follows: 'The DSKPDF scheme of the UGC was an outstanding initiative for enhancing postdoctoral opportunities in India. Over the years the scheme has been administered with remarkable efficiency benefitting a very large number of good researchers. I have personally benefitted by having several co-workers on the DSKPDF scheme. It is imperative to enhance and strengthen the scheme'. We hope that this scheme is strengthened further and extended to other areas of knowledge such as humanities and social sciences.

ACKNOWLEDGEMENTS. We thank the authorities of UGC, New Delhi and SPPU, Pune for support and entrusting the selection under the DSKPDF scheme to the DSKPDF Cell. The guidance and constant support from the past and present EC members is acknowledged. We thank the BSR section of the UGC and all the distinguished referees for their support to run the scheme effectively.

Received 8 June 2019; revised accepted 26 November 2019

doi: $10.18520 / \mathrm{cs} / \mathrm{v} 118 / \mathrm{i} 3 / 352-355$ 